\title{
On the Quasi-Periodic Oscillations of Magnetars
}

\author{
A. Colaiuda ${ }^{1 \star}$, H. Beyer ${ }^{2} \dagger$ and K. D. Kokkotas ${ }^{1,3} \ddagger$ \\ ${ }^{1}$ Theoretical Astrophysics, University of Tübingen, Auf der Morgenstelle 10, 72076, Tübingen, Germany \\ 2 Instituto de Fisica y Matematicas, Universidad Michoacana de San Nicolas de Hidalgo Edificio C-3, \\ Ciudad Universitaria Morelia, Michoacan C.P. 58040, Mexico \\ ${ }^{3}$ Department of Physics, Aristotle University of Thessaloniki, Thessaloniki 54124, Greece
}

20 November 2018

\begin{abstract}
We study torsional Alfvén oscillations of magnetars, i.e., neutron stars with a strong magnetic field. We consider the poloidal and toroidal components of the magnetic field and a wide range of equilibrium stellar models. We use a new coordinate system $(X, Y)$, where $X=\sqrt{a_{1}} \sin \theta, Y=\sqrt{a_{1}} \cos \theta$ and $a_{1}$ is the radial component of the magnetic field. In this coordinate system, the $1+2$-dimensional evolution equation describing the quasi-periodic oscillations, QPOs, see Sotani et al. (2007), is reduced to a $1+1$-dimensional equation, where the perturbations propagate only along the $Y$-axis. We solve the $1+1$-dimensional equation for different boundary conditions and open magnetic field lines, i.e., magnetic field lines that reach the surface and there match up with the exterior dipole magnetic field, as well as closed magnetic lines, i.e., magnetic lines that never reach the stellar surface. For the open field lines, we find two families of QPOs frequencies; a family of "lower" QPOs frequencies which is located near the $X$-axis and a family of "upper" frequencies located near the $Y$-axis. According to Levin (2007), the fundamental frequencies of these two families can be interpreted as the turning points of a continuous spectrum. We find that the upper frequencies are constant multiples of the lower frequencies with a constant equaling $2 n+1$. For the closed lines, the corresponding factor is $n+1$. By these relations, we can explain both the lower and the higher observed frequencies in SGR 1806-20 and SGR $1900+14$.
\end{abstract}

Key words: relativity - MHD - stars: neutron - stars: oscillations - stars: magnetic fields - gamma rays: theory

\section{INTRODUCTION}

Strongly magnetized compact stars, so called 'magnetars', reveal their presence via giant flares with peak luminosities of $10^{44}-10^{46} \mathrm{ergs} / \mathrm{s}$. The giant flares are typically accompanied by a decaying tail which may last several hundreds of seconds. These sources are known as Soft Gamma Repeaters (SGRs), and up to now, three giant flares have been detected, SGR 0526-66 in 1979, SGR 1900+14 in 1998, and SGR 1806-20 in 2004. The analysis of the timing of the last two events revealed several QPOs in the decaying tail whose frequencies are approximately 18, 26, 30, 92, 150, 625, and $1840 \mathrm{~Hz}$ for SGR 1806-20, and 28, 53, 84, and $155 \mathrm{~Hz}$ for SGR 1900+14, see Watts \& Strohmayer (2006). It is believed that during an SGR event torsional oscillations in the solid crust of the star could be excited, (Duncan 1998), that

\footnotetext{
* E-mail:colaiuda@tat.physik.uni-tuebingen.de

$\dagger$ E-mail:horst@cct.lsu.edu

$\ddagger$ E-mail:kostas.kokkotas@uni-tuebingen.de
}

lead to the observed frequencies in the X-ray tail. Indeed, the frequency of many of these oscillations fit the values of the torsional mode oscillations of the solid crust of a compact star. However, since not all of the observed frequencies are explainable by pure crust oscillations, Sotani et al. (2007); Samuelsson \& Andersson (2007), alternative scenarios have also been suggested. For example, Glampedakis et al. (2006) claimed that the observed spectra may be explained via Alfvén oscillations, while Levin (2006) pointed out that the Alfvén oscillations of magnetars could be continuous. Recently, a toy model calculation by Levin (2007) indicated that the edges or turning points of the continuum could correspond to long-lived QPOs, and according to Levin, the results of Sotani et al. (2008a) indicated for realistic magnetar configurations that the Alfvén oscillations of magnetars could be continuous which would explain the lower observed frequencies. In a more recent paper by Sotani \& Kokkotas (2009), it was shown that the spectrum of the polar Alfvén oscillations is actually discrete. 
In Sotani et al. (2008a), the authors performed twodimensional numerical simulations of linearized Alfvén oscillations in magnetars. Their model improves the previously considered toy models in various ways. General Relativity is assumed, various realistic equations of state (EOS) are considered and a consistent dipolar magnetic field is constructed. However, it does not take into account the presence of a solid crust and only examines the response of the ideal magnetofluid to a chosen initial perturbation. The twodimensional partial differential equation (PDE) that was used to study the Alfvén oscillations has a mathematical pathological behavior, as will explained later, that forced them to introduce artificial numerical viscosity in an attempt to stabilize the numerical evolution. The numerical results presented in Sotani et al. (2008a) are compatible with the observations. For example, they found two families of QPOs, corresponding to the edges or turning points of a continuum, with harmonics at near integer multiples. With this identification, they could set an upper limit to the dipole magnetic field of $\sim 3$ to $7 \times 10^{15} \mathrm{G}$, and they could limit the models to very stiff EOS for values of the magnetic field strength near to its upper limit, or moderately stiff for lower values of the magnetic field.

In an extension of that paper, Sotani et al. (2008b), studied the axisymmetric crust torsional modes of magnetars with poloidal and toroidal magnetic field, where both components are confined in the crust. The numerical results showed that this magnetic configuration cannot explain the actual observational data of SGRs, and is in agreement with a more recent result by van Hoven \& Levin (2008).

After a more elaborate study of the master equation, we discovered the origin of the numerical instability, and, moreover, we managed to reduce it, by using a coordinate transformation, to a $1+1$-dimensional PDE which can be evolved stably without the need of artificial viscosity and also allows a semi-analytically estimate of the properties of the continuum and the associated QPO's.

In this paper, we adopt the units of $c=G=1$, where $c$ and $G$ denote the speed of light and the gravitational constant, respectively, and the metric signature is $(-,+,+,+)$.

\section{THE EQUATION OF THE PROBLEM}

We consider a spherically symmetric and static star described by the TOV equations and the line element

$$
d s^{2}=-e^{2 \Phi(r)} d t^{2}+e^{2 \Lambda(r)} d r^{2}+r^{2}\left(d \theta^{2}+\sin ^{2}(\theta) d \phi^{2}\right) .
$$

The structure of the star is not influenced by the presence of the magnetic field because the last has an energy $E_{m}$ which is a few orders of magnitude smaller than the gravitational binding energy $E_{g}$. Typically, $E_{m} / E_{g} \simeq 10^{-4}\left(B / 10^{16} G\right)^{2}$.

Generally, spherical stars show two type of oscillations, spheroidal oscillations with polar parity and toroidal oscillations with axial parity. The observed QPOs in SGR Xray tails may originate from toroidal oscillations, since these can be excited more easily than poloidal oscillations because they do not involve density variations.

The MHD oscillations of the equilibrium model are described by the linearized equation of motion and by the magnetic induction equations, see Sotani et al. (2007). These two perturbation equations can be converted into a $1+2$ dimensional evolution equation for the displacement function $\mathcal{Y}(t, r, \theta)$ which is related to the contravariant component of the perturbed 4 -velocity, $\delta u^{\phi}$ via

$$
\delta u^{\phi}=e^{-\Phi} \partial_{t} \mathcal{Y}(t, r, \theta) .
$$

For an analytical derivation, see Sotani et al. (2008a). The $1+2$-dimensional time-evolution equation for the displacement $\mathcal{Y}(t, r, \theta)$ has the following form (Sotani et al. (2008a) ) :

$A_{t t} \frac{\partial^{2} \mathcal{Y}}{\partial t^{2}}=A_{20} \frac{\partial^{2} \mathcal{Y}}{\partial r^{2}}+A_{11} \frac{\partial^{2} \mathcal{Y}}{\partial r \partial \theta}+A_{02} \frac{\partial^{2} \mathcal{Y}}{\partial \theta^{2}}+A_{10} \frac{\partial \mathcal{Y}}{\partial r}+A_{01} \frac{\partial \mathcal{Y}}{\partial \theta}$

All the coefficients $A_{t t}, A_{20}, A_{11}, A_{02}, A_{10}$ and $A_{01}$ depend on the coordinates $r$ and $\theta$, but not on time. They are given by

$$
\begin{aligned}
& A_{t t}=\left[\epsilon+\rho+\frac{a_{1}^{2}}{\pi r^{4}} \cos ^{2}(\theta)+\frac{a_{1}^{\prime 2}}{4 \pi r^{4}} e^{-2 \Lambda} \sin ^{2}(\theta)\right] e^{-2(\Phi-\Lambda)} \\
& A_{20}=\frac{a_{1}^{2}}{\pi r^{4}} \cos ^{2}(\theta)+\mu \\
& A_{11}=-\frac{a_{1} a_{1}^{\prime}}{\pi r^{4}} \cos (\theta) \sin (\theta) \\
& A_{02}=\frac{a_{1}^{\prime 2}}{4 \pi r^{4}} \sin ^{2}(\theta)+\frac{\mu}{r^{2}} e^{2 \Lambda} \\
& A_{10}=\left(\Phi^{\prime}-\Lambda^{\prime}\right) \frac{a_{1}^{2}}{\pi r^{4}} \cos ^{2}(\theta)+\frac{a_{1} a_{1}^{\prime}}{2 \pi r^{4}} \sin ^{2}(\theta)+\left[\mu^{\prime}+\mu\left(\frac{4}{r}-\Lambda^{\prime}+\Phi^{\prime}\right)\right]
\end{aligned}
$$

$A_{01}=\left[\frac{a_{1}}{\pi r^{4}}\left(2 \pi j_{1}-\frac{a_{1}}{r^{2}}\right)+3 \frac{a_{1}^{\prime 2}}{4 \pi r^{4}}\right] \sin (\theta) \cos (\theta)+\frac{3}{r^{2}} \mu \cot (\theta) e^{2 \Lambda}$,

where $a_{1}(r)$ is the radial component of the electromagnetic four-potential and $j_{1}(r)$ is the four-current. A prime indicates a derivative with respect the radius. The symbol $\mu$ denotes the shear modulus. In the following, we will set $\mu$ equal to zero, neglecting the presence of a solid crust, since we will focus on the oscillations of the magneto-fluid core that plays a major role in magnetars QPOs. In fact, as it has been shown by Levin (2006) and Glampedakis et al. (2006), in the presence of a strong magnetic field pure crustal modes cannot explain the present QPOs frequencies. As a consequence, it is necessary to study global modes, i.e., Alfvén waves propagating through the whole star.

The radial function $a_{1}$ is determined by solving the Grad-Shavranov equation:

$$
a_{1}^{\prime \prime} e^{-2 \Lambda}+\left(\Phi^{\prime}-\Lambda^{\prime}\right) e^{-2 \Lambda} a_{1}{ }^{\prime}-\frac{2}{r^{2}} a_{1}=-4 \pi j_{1} .
$$

As boundary conditions, we assume regularity at the center, $a_{1} \simeq \alpha_{0} r^{2}$, where $\alpha_{0}$ is a constant, and continuity at the surface with a dipole field outside the star.

According to Sotani et al. (2007), the solutions of equation (3) have to satisfy the following boundary conditions:

- regularity at the center: $\mathcal{Y}=0$ at $r=0$;

- no traction on the surface: $\mathcal{Y}_{{ }_{r}}=0$ at $r=R$;

- axisymmetry: $\mathcal{Y}_{, \theta}=0$ at $\theta=0$;

- equatorial plane symmetry for $\ell=2$ initial data: $\mathcal{Y}=0$ at $\theta=\pi / 2$;

- for $\ell=3$ initial data: $\mathcal{Y}_{, \theta}=0$ at $\theta=\pi / 2$. 


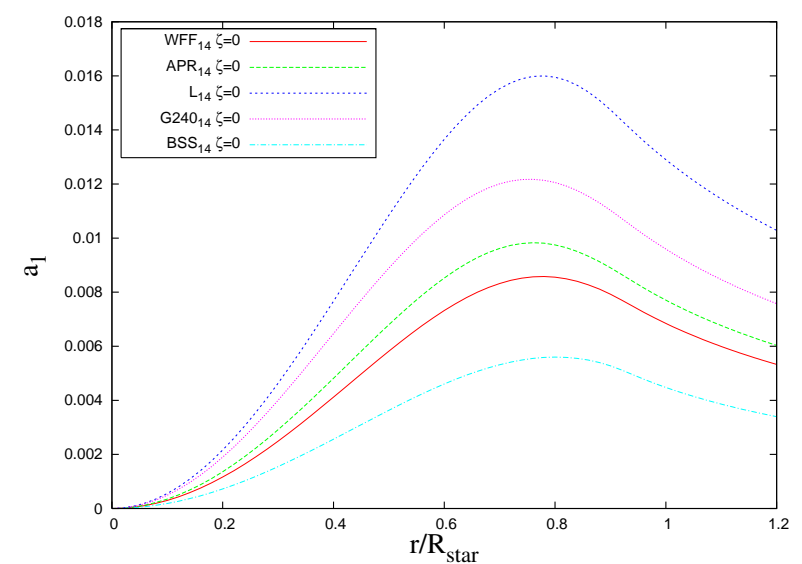

Figure 1. Plot of $a_{1}(r)$ for different EOS. The coordinate $r$ is normalized to the stellar radius.

In Sotani et al. (2008a), in order to avoid numerical instability, equation (3) was solved by introducing an artificial 4-th order 'dissipation' term. Here, instead, we find a coordinate transformation that allows us to avoid the use of a dissipation term and, as we will show later, to avoid the singular behavior of the time independent form of the equation (3). In fact, if, in a first approximation, we neglect the first derivatives in the direction of $r$ and $\theta$ in the equation (3), then only the coefficients $A_{20}, A_{11}$ and $A_{02}$ remain. Calculating the determinant of the principal part of the equation (3), we find that

$$
\begin{aligned}
D & =A_{20} A_{02}-\left(\frac{A_{11}}{2}\right)^{2} \\
& =\frac{a_{1}^{2} a_{1}{ }^{\prime 2}}{4 \pi^{2} r^{8}} \cos ^{2}(\theta) \sin ^{2}(\theta)-\frac{a_{1}^{2} a_{1}{ }^{\prime 2}}{4 \pi^{2} r^{8}} \cos ^{2}(\theta) \sin ^{2}(\theta)=0 .
\end{aligned}
$$

Hence the equation is parabolic in every point of its domain, and therefore does not describe propagation of $2 \mathrm{D}$ waves. Significant simplification is achieved by introduction of a new coordinate system given by the transformation

$$
X=\sqrt{a_{1}} \sin \theta \quad Y=\sqrt{a_{1}} \cos \theta .
$$

This new coordinates system is a kind of Cartesian coordinate system where, instead of the radial coordinate $r$, we use the radial component of the magnetic four-potential $a_{1}$. The behavior of $a_{1}$ inside the star is determined via the equation (10). On the surface, we require the continuity of $a_{1}$ with a dipole solution outside. In Figure 1 we plot the function $a_{1}(r)$ for different equations of state (EOS). The subscripts indicate the mass of the star. For example, $\mathrm{WFF}_{14}$ corresponds to an equilibrium stellar model with EOS WFF (Wiringa et al. (1988)) and $M=1.4 M_{\odot}$.

The magnetic field $B^{\alpha}$ and the radial function $a_{1}$ are related by

$$
B^{\alpha}=\epsilon^{\alpha \beta \mu \nu} u_{\beta} F_{\mu \nu},
$$

where $\epsilon^{\alpha \beta \mu \nu}$ is the Levi-Civita tensor, $u_{\beta}$ is the background four-velocity and $F_{\mu \nu}$ is the electromagnetic tensor. Then equation (13) leads to

$$
H_{r}=\frac{a_{1} e^{\Lambda}}{\sqrt{\pi} r^{2}} \cos \theta \quad H_{\theta}=\frac{a_{1}^{\prime} e^{-\Lambda}}{\sqrt{4 \pi}} \sin \theta
$$

where we define $H_{\alpha}:=B_{\alpha} / \sqrt{4 \pi}$. Note, however, that the coordinates defined by (12) are not the usual magnetic coordinates known in the literature, see for example Alladio et al. (1996) for a review, since they mix the poloidal angle and the radial component of the magnetic field in $(X, Y)$.

When we transform equation (3) according to (12), we get:

$$
\begin{aligned}
& \pi r^{4} A_{t t} \frac{\partial^{2} \mathcal{Y}}{\partial t^{2}}=\frac{1}{4} a_{1} a_{1}{ }^{\prime 2} \frac{\partial^{2} \mathcal{Y}}{\partial Y^{2}}+\frac{y}{2} a_{1}\left(a_{1}{ }^{\prime \prime}-\frac{a_{1}{ }^{\prime 2}}{2}\right) \frac{\partial \mathcal{Y}}{\partial Y} \\
& +Y\left\{\frac{a_{1}{ }^{\prime}}{2}(\Phi-\Lambda)^{\prime} Y^{2}-X^{2}\left[\left(2 \pi j_{1}-\frac{a_{1}}{r^{2}}\right) e^{2 \Lambda}+\frac{a_{1}{ }^{\prime \prime}}{2}\right]\right\} \frac{\partial \mathcal{Y}}{\partial Y} \\
& +X Y^{2}\left\{\frac{a_{1}^{\prime}}{2}(\Phi-\Lambda)^{\prime}+\left[\left(2 \pi j_{1}-\frac{a_{1}}{r^{2}}\right) e^{2 \Lambda}-\frac{a_{1}{ }^{\prime \prime}}{2}\right]\right\} \frac{\partial \mathcal{Y}}{\partial X} .
\end{aligned}
$$

By substituting $a_{1}$ " from equation (10) into the second and third term on the right hand side of equation (15), we arrive at

$$
A_{t t} \frac{\partial^{2} \mathcal{Y}}{\partial t^{2}}=\tilde{A}_{10} \frac{\partial^{2} \mathcal{Y}}{\partial Y^{2}}+\tilde{A}_{01} \frac{\partial \mathcal{Y}}{\partial Y}
$$

where

$$
\begin{aligned}
& \tilde{A}_{10}=\frac{1}{4 \pi r^{4}} a_{1} a_{1}^{\prime 2} \\
& \tilde{A}_{01}=\frac{X}{2 \pi r^{4}} a_{1}\left(\frac{2}{r^{2}} a_{1} e^{2 \Lambda}-4 \pi j_{1} e^{2 \Lambda}-\frac{a_{1}{ }^{2}}{2}\right),
\end{aligned}
$$

i.e., the coefficient of $\partial \mathcal{Y} / \partial X$ vanishes identically. The equation (16) is a $1+1$-dimensional wave equation. The perturbations propagate only along the $Y$ direction. In the last direction, the problem reminds of a study of waves propagating along the strings of a musical instrument.

The construction of the grid in these $(X, Y)$ coordinates needs special attention. The function $a_{1}$ has a maximum inside the star, and the position of this maximum depends on the strength and the topology of the magnetic field. A strong magnetic field, and also a toroidal field added to the standard poloidal field, pushes the maximum near the surface of the star. The position of $a_{1}$ max determines also the number of the closed magnetic field lines confined inside the star. Since the closed magnetic field lines don't reach the surface, they are subject to different boundary conditions than the open magnetic field lines. This difference leads to a different behaviour of the oscillations of open and closed magnetic lines, as will be shown later. In practice, we split the problem into two parts for both cases, i.e., for closed and open magnetic field lines. For the open magnetic field lines, the perturbation function $u(X, Y)$ is evolved until some points $X_{\max }$ and $Y_{\max }$ that correspond to the maximum of $a_{1 \text { max }}$, i.e., we work on the part $A$ of Figure 2 Then the evolution continues, in the part $A_{1}$ of Figure 2, from the points $X_{\max }$ and $Y_{\max }$ and ends at the points $X_{\text {surf }}$ and $Y_{\text {surf }}$ that correspond to the surface of the star. Note that in this second part, we have to use the transformation

$$
X=-\sqrt{a_{1}} \sin \theta \quad Y=-\sqrt{a_{1}} \cos \theta,
$$

due to the sign change of the derivative of $a_{1}$. In a similar fashion for the closed magnetic lines, we evolve $u(X, Y)$ until $X_{\max }$ and $Y_{\max }$, but then the simulation doesn't end on the surface, but at $X_{\text {eq }}$ and $Y_{\text {eq }}$, that correspond to points on the equator. From a technical point of view, we just "open" the field lines, then we solve the two problems separately 


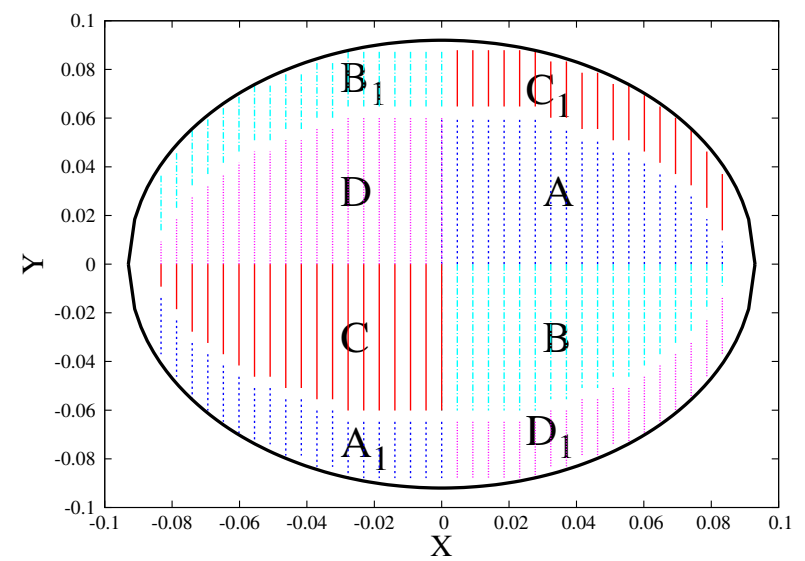

Figure 2. Plot of the magnetic field lines in the $(X, Y)$ coordinates. Note that, after the point $X\left(a_{1} \max \right)$ and $Y\left(a_{1} \max \right)$, lines of the same color continue in different quadrants. For example, the lines of the domain $A$ continue in the domain $A_{1}$.

and match the two solutions that we get via the boundary conditions. This procedure will be explained later. Figure 2 may help in understanding the procedure followed in solving this problem. The equation (16) must be solved with the appropriate boundary conditions. The boundary condition are different for open and closed magnetic lines. The open lines reach the surface and match up with the dipole magnetic field outside the star. Then, as we will show later, they couple on the surface and oscillate together. Differently, the closed magnetic lines confine themselves in the interior of the star, forming closed loops, and they never reach the surface. So they don't have any coupling to the open magnetic lines and oscillate independently. When we 'open' the closed magnetic lines, creating magnetic strings, we open the closed loop, but don't change the boundary conditions at the ends of the strings.

In the $(X, Y)$ coordinate system, the boundary conditions become

- regularity at the center: $\mathcal{Y}(X, Y)=0$ for $X=0$ and $Y=0$,

- no traction on the surface for the open lines:

$$
\frac{a_{1}{ }^{\prime}}{2 a_{1}}\left[X \frac{\partial \mathcal{Y}}{\partial X}+Y \frac{\partial \mathcal{Y}}{\partial Y}\right]=0
$$

- axisymmetry at $X=0$ :

$$
Y \frac{\partial \mathcal{Y}}{\partial X}=0
$$

- equatorial plane symmetry for $\ell=2$ initial data: $\mathcal{Y}(X, Y)=0$ at $Y=0$.

- for $\ell=3$ initial data at $Y=0$ :

$$
Y \frac{\partial \mathcal{Y}}{\partial X}=0
$$

As a consequence, for initial data with $\ell=2$, the open 'magnetic strings' are coupled on the surface of the star and near the axis for $X=0$ (corresponding to $\theta=0$ ). With initial data $\ell=3$, there is an additional coupling at the equator (corresponding to $Y=0$ ).

\section{$2.1 \quad$ Numerical method}

We study a variety of equilibrium models, constructed for three representative EOSs and different masses that range from $1.4-2.0 M_{\odot}$, see Table 1] We set the magnetic field strength to $B_{\mu}=4 \times 10^{15}$ Gauss, matching the field strength extrapolated from the analysis of the observations for SGR $1806-20$ and SGR $1900+14$. We construct a numerical equidistant grid $90 \times 90$ in the $(X, Y)$ coordinates, setting $X_{\max }=\sqrt{a_{1 \max }} \sin \theta$ and $Y_{\max }=\sqrt{a_{1 \max }} \cos \theta$ and varying $\theta$ from 0 and $\pi / 2$. The structure of our grid is displayed in the first quadrant of Figure 2. Note that each 'magnetic string' in the first quadrant continues in the third quadrant. We evolve these two parts in time like two separate problems, taking into account the different boundary conditions for open and closed magnetic field lines. The two parts are linked in every time step, requiring the continuity of the function $\mathcal{Y}$ and its derivative $\mathcal{Y}^{\prime}$. For our evolution, we use an iterative Crank-Nicholson scheme and we evolve the perturbations for more than 1s (see Figure 3). In order to check the stability of the scheme, we performed an evolution for $2 \mathrm{~s}$ and found that our scheme was still stable.

We evolve the perturbations by providing initial data corresponding to either odd, $\ell=3$ or even, $\ell=2$, boundary conditions at $Y=0$. A change in the value of $\ell$ leads to different boundary conditions for open and closed lines and leads to different scaling in the oscillation frequencies of the open field lines.

We compute the FFT of various 'strings' and find that each 'string' corresponds to a different frequency. In Sotani et al. (2008a), the authors find a family of upper frequencies near the magnetic axis for $\theta=0$, corresponding to points along the $\mathrm{Y}$-axis in our coordinates, and a family of lower frequencies for $\theta=\pi / 2$, corresponding to points along the $\mathrm{X}$-axis in our coordinates. They identify the fundamental frequency peaks of the two families of QPOs with the edges or turning points of the Alfvén continuum suggested by Levin (2007). These two frequencies were named fundamental lower $L_{0}$, for $\theta=\pi / 2$, and fundamental upper $U_{0}$, for $\theta=0$ QPOs. The overtones are indicated by $L_{n}$ and $U_{n}$. In the following, we adopt these conventions when we refer to the oscillations of the open field lines, while we call the frequencies of the closed field lines $C_{n}$.

Our results confirm partially the results of Sotani et al. (2008a). For open lines, a family of upper frequencies appears near the $\mathrm{Y}$-axis, while a family of lower frequencies appears near the last open magnetic field line, just before the region of closed lines. This can be explained by the fact that the 'strings' near the $\mathrm{Y}$-axis correspond to a stronger average magnetic field because in the $(r, \theta)$ coordinates they correspond to denser field lines, see Figure 6 Since the frequency of the Alfvén oscillations is proportional to the strength of the magnetic field, we expect that these frequencies are the upper ones. In addition, we find that, for all the 'magnetic strings', the upper frequencies are multiples of the fundamental frequency both in the case of $L_{n}$ and in the case of $U_{n}$, see Figure 4 However, this multiplicity is different when we consider even $\ell=2$ or odd $\ell=3$, initial data. In fact, we obtain

$f_{L_{n}^{\text {even }}} \simeq(2 n+1) f_{L_{0}}$

$f_{U_{n}^{\text {even }}} \simeq(2 n+1) f_{U_{0}}$ 
$f_{L_{n}^{\text {odd }}} \simeq(n+1) f_{L_{0}}$

$f_{U_{n}^{\text {odd }}} \simeq(n+1) f_{U_{0}}$

where the superscripts even and odd indicate results derived for even $\ell=2$ and odd $\ell=3$ parity initial data and boundary conditions on the equator. The different scaling shown in the relations (23) - (24) and (25)-(26) is due to the different boundary conditions used for odd and even values of $\ell$.

The problem can be understood by looking at a simple problem of two strings of finite length connected by by another spring. If the two strings are connected at just one of their ends, while the others are kept fixed, like in the case of even parity where the 'magnetic strings' are coupled just near the surface and are fixed at the equator, then we obtain the relations (23) and (24). This type of configuration was analyzed in Kokkotas \& Schutz (1986), in order to explain the w-modes of neutron stars. An analytic derivation of the relations (23) and (24) can be found there. If instead the two strings are coupled in both ends, like in the case of odd parity, then the relations (25) - (26) follow.

The frequencies of the closed magnetic field lines show a different behaviour. The upper and lower frequencies are scaling as, see also Figure 5]

$$
f_{C_{n}} \simeq(n+1) f_{C_{0}} .
$$

The difference between the relation (23) and (27) is due to the different boundary conditions. The closed lines never reach the surface. They start from the equator where they close. This means that they are subject to the same boundary condition at both of their ends which resembles the toy problem of a string that oscillates with both ends fixed and gives the $n+1$ behaviour, as in the case odd values of $\ell$ for the open field lines. The frequencies that we find for closed lines initially decrease to a minimum and from then on increase. This behaviour results from two factors. First, from the fact that closed lines are shorter than open lines, and, second, from the fact that the magnetic field becomes somehow locally weaker as we move off the axis. This behaviour of the spectrum has been identified after comparing our data with the results coming from the non-linear evolutions presented in Cérda-Durán et al. (2009).

The relations here provide the limits of the continuous part of the spectrum, in accordance with the results of Levin (2007). Our results are summarized in Table 1 .

\subsection{Why we don't need artificial viscosity}

In the preceding work Sotani et al. (2008a), an artificial viscosity was added in order to avoid a fast growing instability. This instability is due to the presence of the so called " $O$ type neutral point" (Taylor (1979)). If the magnetic field lines of a poloidal magnetic field are closed and confined to the stellar interior, then there is a point on which these magnetic field lines converge and become degenerate (see Figure 6). This point corresponds to the maximum of the function $a_{1}$.

In our scheme, the magnetic field lines are practically straight, see Figure 2 even in the vicinity of the point that corresponds to $a_{1} \max$. This behavior is due to our choice to 'open' the grid and to evolve the open and closed field lines as two independent problems. When we look at the point

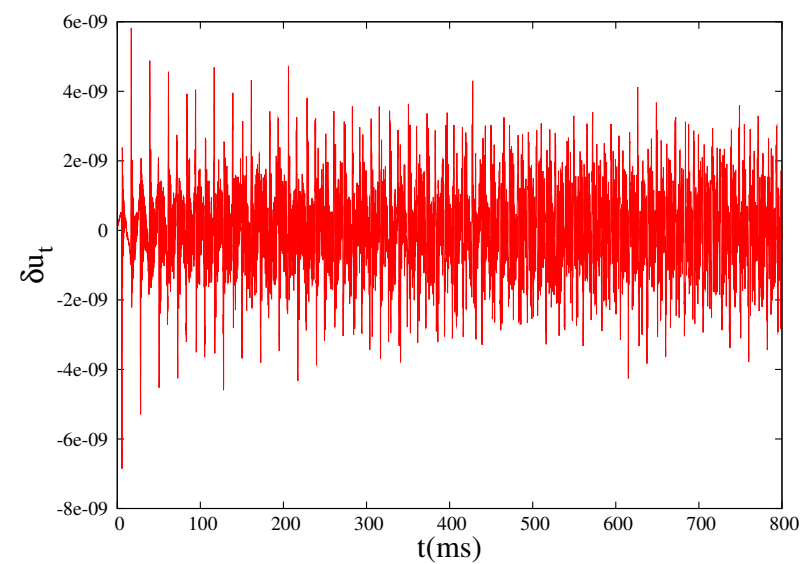

Figure 3. The time evolution of $\partial_{t} \mathcal{Y}$ for an open line in a point near the $\mathrm{Y}$-axis for the stellar equilibrium model $\mathrm{WFF}_{14}$ and $\zeta=0$.

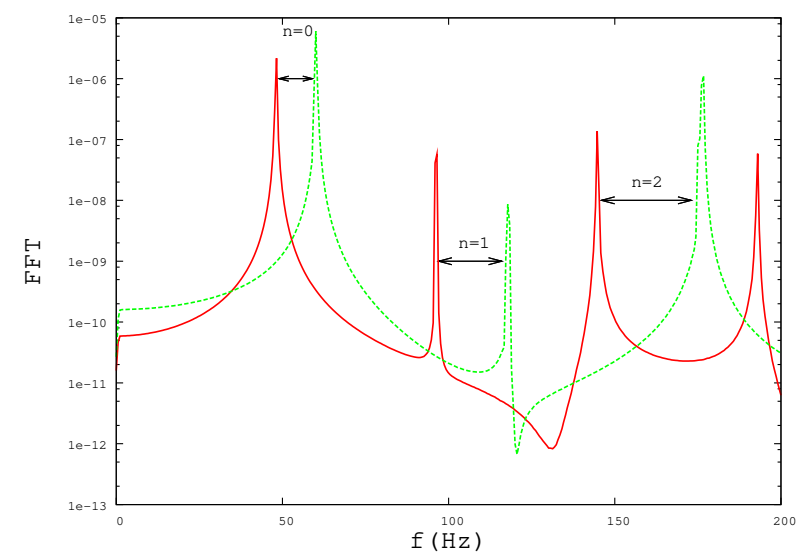

Figure 4. The FFT of a point near the $\mathrm{Y}$-axis, green/dashed line, and near the critical point, red/continuous line, for open lines and even parity, $\ell=2$. The stellar model is $\mathrm{WFF}_{14}$.

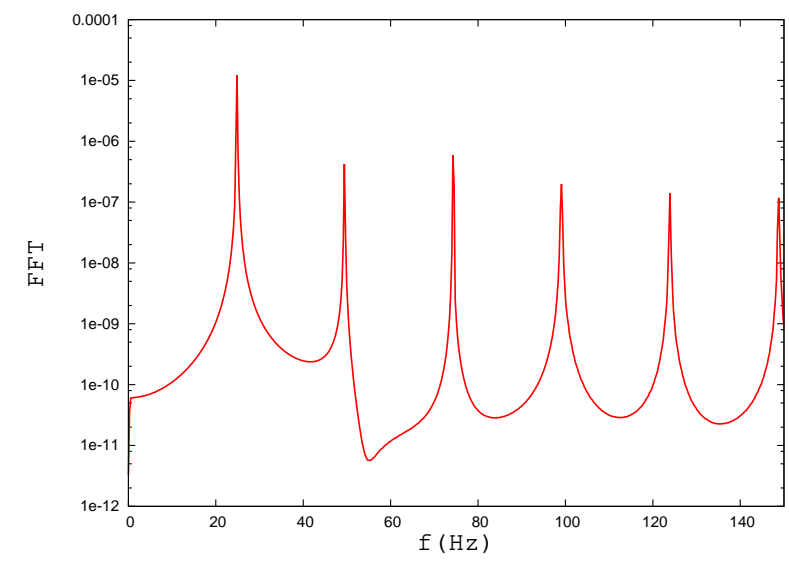

Figure 5. The FFT of the first closed line for the stellar equilibrium model $\mathrm{WFF}_{14}$. It is worth noticing the spacing of the peaks in accordance to (27) 


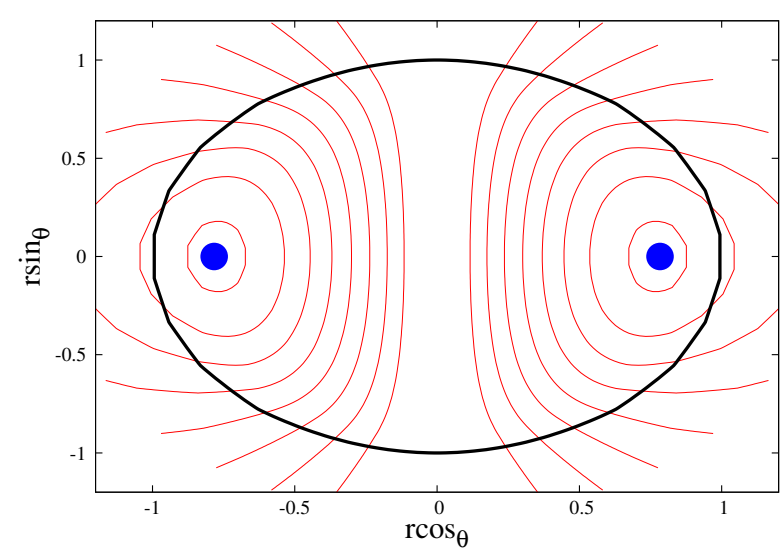

Figure 6. The magnetic field lines for the equilibrium stellar model $\mathrm{WFF}_{14}$ and $\zeta=0$.

$a_{1 \text { max }}$, we find that it practically doesn't evolve and, because the 'strings' are coupled only along the surface and near the $X=0$ axis, cannot be influenced by the oscillations of the other 'strings'. In this way, we avoid the singular behaviour shown in the $(r, \theta)$ coordinates. The last is the main reason why our code is stable also without artificial viscosity.

\subsection{Toroidal fields}

From equation (13), the toroidal magnetic field can be written as

$$
H_{\phi}=-\frac{\zeta e^{-\phi} a_{1}}{\sqrt{4 \pi}} \sin \theta,
$$

where $\zeta$ is a constant only depending on the toroidal magnetic field. By increasing or decreasing $\zeta$, we can influence the strength of the toroidal magnetic field. So $\zeta$ can be interpreted as a parameter that describes the strength of the toroidal magnetic field in relation to the poloidal field, see Colaiuda et al. (2008) for details. The presence of a toroidal field modifies equation (10) by adding an extra term $\zeta^{2} e^{-2 \nu} a_{1}$ (see Sotani et al. (2008b)).

$a_{1}^{\prime \prime} e^{-2 \Lambda}+(\Phi+\Lambda)^{\prime} e^{-2 \Lambda} a_{1}{ }^{\prime}+\left(\zeta^{2} e^{-2 \Phi}-\frac{2}{r^{2}}\right) a_{1}=-4 \pi j_{1}$.

Hence, the toroidal field influences our evolution equation (16) only through the presence of the extra term $a_{1}{ }^{\prime \prime}$. By solving equation (29) for $a_{1}^{\prime \prime}$ and subsequent substitution into equation (16), the toroidal magnetic field appears in the $1+1$ equation (16) only through the parameter $\zeta$ in the term

$$
\tilde{A}_{01}=\frac{X}{2} a_{1}\left(\frac{2 e^{2 \Lambda} a_{1}}{r^{2}}-\zeta^{2} a_{1} e^{-2 \Phi}-\frac{a_{1}{ }^{2}}{2}-4 \pi j_{1}\right) .
$$

This means that the toroidal field affects the local propagation speed of the Alfvén modes. Also, the toroidal field pushes the position of $a_{1}$ max outwards and contributes to the stability of the magnetic field in the star. In Figure 7 we plot the function $a_{1}$ for the EOS $\mathrm{WFF}_{14}$ and for different values of the parameter $\zeta$. It is clear that when $\zeta$ increases, i.e., the strength of the toroidal magnetic field increases, then $a_{1 \text { max }}$ is pushed closer to the surface .

Hence, it is natural to expect changes in the oscillation

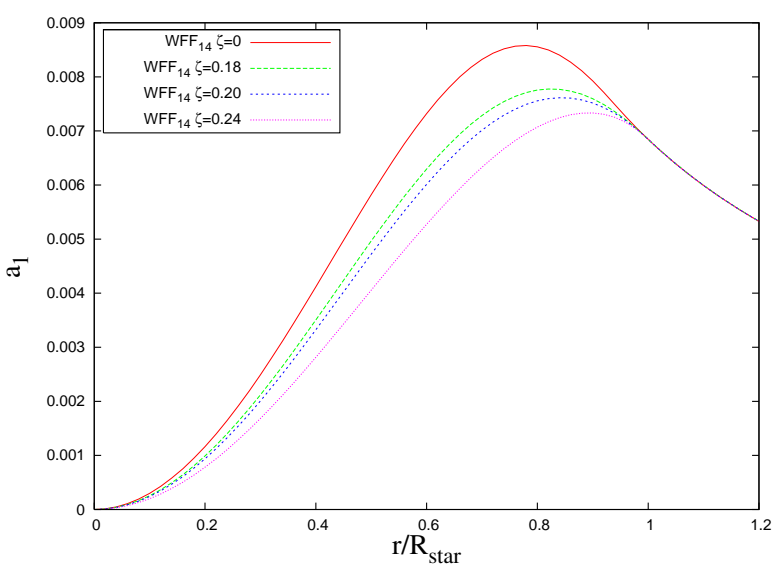

Figure 7. The function $a_{1}$ as a function of the normalized coordinates $r / R$ for an equilibrium model constructed with the equation of state $\mathrm{WFF}$ and a mass $M=1.4 M_{\odot}$. Different curves reflect different values of the parameter $\zeta$, i.e., different strength of the toroidal magnetic field.

spectrum due to the presence of the toroidal component of the magnetic field. We find that the frequencies are lower compared to the case in which only a poloidal field is present, see Table 2 especially for the closed magnetic 'strings'. This can be explained because, by the presence of a toroidal magnetic field $a_{1 \mathrm{max}}$, i.e., the point were the magnetic field lines converge, moves outwards, and hence the 'magnetic strings' increase in length. Since the frequency is inversely proportional to the length of the 'strings', as the length increases, the frequency decreases.

\section{IDENTIFICATION OF THE QPOS FREQUENCIES}

In a paper by Watts \& Strohmayer (2006), the timing analysis of the SGR 1806-20 and the SGR 1900+14 has been computed. The study shows the appearance of several QPOs in the tail of these events. In particular, for SGR 1806-20, the identified frequencies are 18, 26, 30, 92, 150, 625 and 1840 $\mathrm{Hz}$, while for SGR $1900+14$, the frequencies are $28,53,84$ and $155 \mathrm{~Hz}$.

Using the relations (25) and (26), we can easily show that for SGR $1900+14$, if we consider $f_{L_{0}^{\text {odd }}}=28 \mathrm{~Hz}$, we can identify $f_{L_{1}^{\text {odd }}}=56 \mathrm{~Hz}$, and $f_{L_{2}^{\text {odd }}} \simeq 84 \mathrm{~Hz}$. In a similar way for SGR 1806-20, we have $f_{U_{0}^{\text {even }}}=18 \mathrm{~Hz}$ and $f_{U_{0}^{\text {odd }}}=30 \mathrm{~Hz}$, and consequently $f_{U_{2}^{\text {even }}} \simeq 92 \mathrm{~Hz}$ or $f_{U_{2}^{\text {odd }}} \simeq 92, f_{U_{4}^{\text {even }}} \simeq$ $150 \mathrm{~Hz}$ or $f_{U_{4}^{\text {odd }}} \simeq 150$. If we look at our results in the Tables 1 and 2, we can identify the QPOs in SGR 1900+14 with the lower frequencies for odd parity of a model with equation of state $\mathrm{WFF}$ and a mass around $1.8 M_{\odot}$. For the QPOs from SGR 1806-20, we can fit the observational data by a model with equation of state $\mathrm{APR}$, mass around $1.4 M_{\odot}$ and a toroidal field a little stronger than the poloidal field , $\zeta=0.18$. 
Table 1. Frequencies of Alfvén QPOs for a representative sample of equilibrium models, constructed for different EOS and fixed magnetic field strength $B=4 \times 10^{15}$ Gauss. The frequencies are computed for points near the critical point, $L_{n}$, and near the Y-axis, $U_{n}$, for open lines, both for even and odd parity, and for the closed line $\left(C_{n}\right)$.

\begin{tabular}{|c|c|c|c|c|c|c|c|}
\hline Model & $M / R$ & $n$ & $f_{L_{n}^{\text {even }}}(H z)$ & $f_{L_{n}^{\text {odd }}}(H z)$ & $f_{U_{n}^{\text {even }}}(H z)$ & $f_{U_{n}^{\text {odd }}}(H z)$ & $f_{C_{n}}(H z)$ \\
\hline \multirow{3}{*}{$W F F_{14}$} & \multirow{3}{*}{0.189} & 0 & 23.00 & 46.56 & 28.05 & 58.90 & 23.56 \\
\hline & & 1 & 69.55 & 93.11 & 84.15 & 118.4 & 47.68 \\
\hline & & 2 & 116.1 & 139.1 & 144.3 & 178.5 & 70.11 \\
\hline \multirow{3}{*}{$W F F_{18}$} & \multirow{3}{*}{0.264} & 0 & 13.46 & 27.48 & 16.27 & 33.09 & 14.02 \\
\hline & & 1 & 39.82 & 54.41 & 48.24 & 66.75 & 28.05 \\
\hline & & 2 & 67.31 & 81.89 & 80.21 & 99.84 & 42.06 \\
\hline \multirow{3}{*}{$A P R_{14}$} & \multirow{3}{*}{0.178} & 0 & 23.01 & 47.06 & 28.24 & 61.18 & 24.04 \\
\hline & & 1 & 70.60 & 95.17 & 86.28 & 121.8 & 74.78 \\
\hline & & 2 & 117.1 & 142.2 & 144.3 & 182.5 & 180.0 \\
\hline \multirow{3}{*}{$A P R_{16}$} & \multirow{3}{*}{0.206} & 0 & 19.04 & 39.68 & 21.69 & 44.97 & 19.57 \\
\hline & & 1 & 58.72 & 79.88 & 66.6 & 89.40 & 39.68 \\
\hline & & 2 & 96.28 & 119.6 & 109.5 & 133.8 & 60.31 \\
\hline \multirow{3}{*}{$A P R_{20}$} & \multirow{3}{*}{0.269} & 0 & 12.53 & 25.06 & 15.25 & 31.60 & 13.07 \\
\hline & & 1 & 37.59 & 50.09 & 44.12 & 61.56 & 25.60 \\
\hline & & 2 & 62.10 & 74.63 & 74.63 & 92.61 & 38.13 \\
\hline \multirow{3}{*}{$L_{20}$} & \multirow{3}{*}{0.198} & 0 & 13.10 & 27.83 & 16.78 & 35.61 & 13.52 \\
\hline & & 1 & 40.11 & 55.66 & 51.16 & 70.40 & 27.85 \\
\hline & & 2 & 68.35 & 83.50 & 85.54 & 105.6 & 41.34 \\
\hline
\end{tabular}

Table 2. Frequencies of Alfvén QPOs and their ratios for different stellar models with a toroidal component of the magnetic field and a surface magnetic field $B=4 \times 10^{15}$ Gauss. For open magnetic 'strings' the frequencies are taken for points near the critical point, $L_{n}$, and near the Y-axis, $U_{n}$, both for even and odd parity. We also give the value of the frequencies for the lower closed magnetic 'string' $\left(C_{n}\right)$.

\begin{tabular}{|c|c|c|c|c|c|c|c|}
\hline Model & $\zeta\left(k m^{-1}\right)$ & $n$ & $f_{L_{n}^{\text {even }}}(H z)$ & $f_{L_{n}^{\text {odd }}}(H z)$ & $f_{U_{n}^{\text {even }}}(H z)$ & $f_{U_{n}^{\text {odd }}}(H z)$ & $f_{C_{n}}(H z)$ \\
\hline \multirow{3}{*}{$W F F_{14}$} & \multirow{3}{*}{0.24} & 0 & 15.15 & 30.30 & 16.24 & 33.94 & 15.76 \\
\hline & & 1 & 46.06 & 61.20 & 50.91 & 66.66 & 31.15 \\
\hline & & 2 & 75.75 & 90.90 & 81.20 & 99.99 & 79.38 \\
\hline \multirow{3}{*}{$A P R_{14}$} & \multirow{3}{*}{0.18} & 0 & 18.95 & 38.46 & 22.85 & 49.6 & 19.51 \\
\hline & & 1 & 56.83 & 76.91 & 69.67 & 98.65 & 39.01 \\
\hline & & 2 & 95.30 & 114.8 & 117.0 & 147.7 & 57.96 \\
\hline \multirow{3}{*}{$A P R_{14}$} & \multirow{3}{*}{0.20} & 0 & 16.21 & 32.42 & 19.35 & 41.84 & 16.73 \\
\hline & & 1 & 48.11 & 64.84 & 59.05 & 83.67 & 33.47 \\
\hline & & 2 & 64.84 & 97.79 & 99.88 & 125.5 & 51.25 \\
\hline \multirow{3}{*}{$L_{14}$} & \multirow{3}{*}{0.20} & 0 & 11.47 & 22.93 & 13.92 & 29.90 & 11.88 \\
\hline & & 1 & 34.40 & 45.46 & 42.18 & 58.57 & 23.75 \\
\hline & & 2 & 56.52 & 68.39 & 70.05 & 88.46 & 35.63 \\
\hline
\end{tabular}

\section{DISCUSSION AND CONCLUSION}

We have investigated torsional Alfvén modes of relativistic stars with poloidal and toroidal magnetic field using a new coordinate system $(X, Y)$, where $X=\sqrt{a_{1}} \sin \theta$ and $Y=\sqrt{a_{1}} \cos \theta$. In this new coordinate system, the perturbed two dimensional equation for the toroidal displacement $\mathcal{Y}(t, r, \theta)$ in Sotani et al. (2008a) is reduced to a $1+1$ dimensional equation, and the perturbations, i.e., Alfvén waves, are propagating only along the Y-direction. By solving the problem with the appropriate boundary conditions for open and closed magnetic 'strings', we find that each 'magnetic string' corresponds to a family of frequencies. The upper frequency belongs to the family of open magnetic 'strings' near the Y-axis because the Alfvén frequency is proportional to the local strength of the magnetic field, and since near the Y-axis, the magnetic field is stronger than in the rest of the star. The family corresponding to the lower 
frequency is the one just before the O-point, i.e., the point where the magnetic lines converge and degenerate into a point. We find that for each family of frequencies there is a relation between the overtone frequencies and the fundamental frequencies, and this relation changes if we consider odd or even parity, see equations (23), (24), (25), (26). In addition, we find a third family of frequencies linked to the closed lines. This family shows a minimum, whose value is similar to that of the lower frequencies of the open lines for even parity. Also, we find that in presence of a toroidal magnetic field the frequencies are lower than in the case with only a dipole magnetic field, both for closed and open magnetic 'string'. This is due to the change in the position of $a_{1}$ max that moves outwards. This results in longer 'magnetic strings' and, as consequence, to a lower characteristic frequency for each 'string'.

In a future work, it will be interesting to study whether the presence of a solid crust can quantitatively and qualitatively alter our results.

\section{ACKNOWLEDGMENTS}

This work has been done in parallel with the work Cérda-Durán et al. (2009), where the same problem has been studied by using non-linear evolutions. We benefited from exchange of ideas and results with the authors and are particularly thankful for collaboration. We are also grateful to Hajime Sotani for helpful comments and for providing us with details of his numerical code. This work was supported via the Transregio 7 "Gravitational Wave Astronomy" financed by the Deutsche Forschungsgemeinschaft DFG (German Research Foundation).

\section{REFERENCES}

Akmal A.,Pandharipande V. R., Ravenhall D. G., 1988, Phys. Rev. C58, 1804

Alladio F., Micozzi P., 1996, Phys.Plasmas, 3, 72

Cérda-Durán P., Stergioulas N., Font J. A., 2009, astro$\mathrm{ph} / 0902.1472$

Colaiuda A., Ferrari V., Gualtieri L., Pons J., 2008, MNRAS, 385, 2080

Duncan R. C.,1998, ApJ, 498, L45

Glampedakis K., Samuelsson L., Andersson N., 2006, MNRAS, 371, L74

Glendenning N. K., 2000, Compact Star, Springer, New York

van Hoven M., Levin Y., 2008, MNRAS, 368, L35

Kokkotas K. D., Schutz B. F.,1986, G.R.G, 18, 913

Levin Y., 2006, MNRAS, 368, L35

Levin Y., 2007, MNRAS, 377159

Levin Y., 2006, MNRAS, 391, 283

Pandharipande V. R., Pines D., Smith A., 1976, Astrophys. J. 208, 550

Samuelsson L., Andersson N., 2007, MNRAS, 374, 256

Sotani H., Kokkotas K. D., Stergioulas N., 2007, MNRAS 375,261

Sotani H., Kokkotas K. D., Stergioulas N., 2008a, MNRAS, $385, \mathrm{~L} 5$
Sotani H., Colaiuda A., Kokkotas K. D., 2008b, MNRAS, 385,2161

Sotani H., Kokkotas K. D., 2009, astro-ph/0902.1490

Tayler R. J. 1980, MNRAS,191, 151

Watts A., Strohmayer T. E., 2006 ApJ, 637, L117

Watts A. L., Strohmayer T. E., 2006, 2006, Adv. Space Res., 40, 1446

Wiringa R. B., Fiks V., Farbrocini A., 1988, Phys. Rev. C38, 1010 